MITSUBISHI ELECTRIC RESEARCH LABORATORIES

http://www.merl.com

\title{
Decoupled Three-Phase Load Flow Method for Unbalanced Distribution Systems
}

\author{
Sun, H.; Dubey, A.; Nikovski, D.; Ohno, T.; Takano, T.; Kojima, Y.
}

TR2012-086 October 2012

\begin{abstract}
This paper proposes a decoupled three-phase load flow analysis method for unbalanced distribution systems. The power flows are solved through nodal current injection mismatch equations written in rectangular coordinates. The voltage changes resulting from nodal current injection mismatches and nodal admittance matrix have been decoupled into one contribution from the real part, conductance matrix, and the other contribution from the imaginary part, susceptance matrix. The method determines voltage changes resulting from conductance and susceptance matrices respectively, and determines the voltages of a node as a linear combination of those voltage changes. The relative contributions are determined based on the diagonals of the conductance and susceptance matrices. The constant active power and voltage magnitude(PV) nodes have been converted into constant active and reactive power(PQ) ones based on a sensitivity matrix determined through Kron reduction of the nodal admittance matrix, and the corresponding reactive powers are adjusted after each solution has converged. The zero- impedance branches are merged with adjacent impedance branches, and the three phases of a balanced-voltage PV bus are merged into one single- phase PV bus to be modeled in the nodal admittance matrix. Numerical examples on IEEE 37 node test feeder and IEEE 123 node test feeder are presented to demonstrate the effectiveness of the proposed method.
\end{abstract}

IEEE International Conference on Power System Technology (POWERCON)

This work may not be copied or reproduced in whole or in part for any commercial purpose. Permission to copy in whole or in part without payment of fee is granted for nonprofit educational and research purposes provided that all such whole or partial copies include the following: a notice that such copying is by permission of Mitsubishi Electric Research Laboratories, Inc.; an acknowledgment of the authors and individual contributions to the work; and all applicable portions of the copyright notice. Copying, reproduction, or republishing for any other purpose shall require a license with payment of fee to Mitsubishi Electric Research Laboratories, Inc. All rights reserved.

Copyright (C) Mitsubishi Electric Research Laboratories, Inc., 2012

201 Broadway, Cambridge, Massachusetts 02139 



\title{
Decoupled Three-Phase Load Flow Method for Unbalanced Distribution Systems
}

\author{
Hongbo Sun, Senior Member, IEEE, Anamika Dubey, Daniel Nikovski, Member, IEEE, Tetsufumi \\ Ohno, Tomihiro Takano, and Yasuhiro Kojima, Member, IEEE
}

\begin{abstract}
This paper proposes a decoupled three-phase load flow analysis method for unbalanced distribution systems. The power flows are solved through nodal current injection mismatch equations written in rectangular coordinates. The voltage changes resulting from nodal current injection mismatches and nodal admittance matrix have been decoupled into one contribution from the real part, conductance matrix, and the other contribution from the imaginary part, susceptance matrix. The method determines voltage changes resulting from conductance and susceptance matrices respectively, and determines the voltages of a node as a linear combination of those voltage changes. The relative contributions are determined based on the diagonals of the conductance and susceptance matrices. The constant active power and voltage magnitude( $P V)$ nodes have been converted into constant active and reactive power( $(P Q)$ ones based on a sensitivity matrix determined through Kron reduction of the nodal admittance matrix, and the corresponding reactive powers are adjusted after each solution has converged. The zero-impedance branches are merged with adjacent impedance branches, and the three phases of a balanced-voltage PV bus are merged into one single-phase $P V$ bus to be modeled in the nodal admittance matrix. Numerical examples on IEEE 37 node test feeder and IEEE 123 node test feeder are presented to demonstrate the effectiveness of the proposed method.
\end{abstract}

Index Terms-- Load flow; Three phase systems; Distribution systems; Decoupled.

\section{INTRODUCTION}

$\mathrm{T}$ HREE phase load flow analysis is a fundamental technique for a distribution management system(DMS) to evaluate and monitor the real time steady-state performance of distribution systems. Various methods for solving three-phase power flow problems are known. These methods differ in the form of the equations describing the system, or in the numerical techniques used. Usually, either topology- or matrix-based methods are employed. Topology-based methods are suitable for radial systems, and include the Backward/Forward sweep and Ladder methods [1]-[2]. Compensation schemes [2]-[4] must be used when loops or constant active power and voltage magnitude (PV) buses are present in the system. The admittance-matrix based methods include the Implicit Z-bus method [5]-[6],

Hongbo Sun, and Daniel Nikovski are with the Mitsubishi Electric Research Laboratories, Cambridge, MA 02139 USA (e-mail: hongbo.sun@merl.com, nikovski@merl.com).

Anamika Dubey is with the University of Texas at Austin, Austin Texas 78712 USA(e-mail:ana.iitr@gmail.com).

Tetsufumi Ohno, Tomihiro Takano, and Yasuhiro Kojima are with the Mitsubishi Electric Corporation, Hyogo 661-8661 Japan (e-mail: Ono.Tetsufumi@ah.MitsubishiElectric.co.jp,Kojima.Yasuhiro@ab.Mitsubi shiElectric.co.jp). the Newton-Raphson method [7]-[8], the Fast Decoupled method [9], and the Sequence Decoupling method [10]. All of these methods have their own limitations when applied to large systems, either in terms of modeling capabilities, or in terms of computational efficiency.

This paper proposes a new decoupled three-phase load flow method. It formulates the power flows as nodal current injection mismatch equations in rectangular coordinates, and decouples the power flow problem into solving conductance matrix contributions and susceptance matrix contributions separately. An effective procedure is provided to obtain the best contribution factors of the two matrices to the final solution. The method requires no additional assumptions regarding decoupling, hence it is much more robust and reliable. It replaces the step of nodal complex admittance matrix factorization with two real matrix factorizations. The matrices are kept constant and require factorization only once, and as a result the method is very robust for a larger range of resistance-to-reactance ratio. The constant active power and voltage magnitude(PV) nodes have converted into constant active and reactive power(PQ) nodes to be modeled. A PV sensitivity matrix method is used to adjust the reactive powers of $\mathrm{PV}$ nodes to maintain required magnitude of voltages. The PV sensitivity matrix is easily obtained by Kron reduction of the nodal admittance matrix to eliminate non-PV nodes. The proposed method explicitly defines zero impedance branches in distribution systems. The method models such branches accurately, and avoids the occurrences of solution divergence that are usually caused by zero impedance branches in conventional methods. By embedding the three-phase balanced-voltage requirements into the nodal admittance matrix, the threephase PV bus model is simplified as a one single-phase model.

\section{Proposed Method}

\section{A. GB Decoupled Three-phase Load Flow}

Using polar coordinates, the power flows can be solved by using the nodal current injection equations:

$$
[I]=[Y][V]
$$

where $[I]$ and $[V]$ are the vector of equivalent complex current injections, and complex voltages for all nodes, and $[Y]$ is the complex nodal admittance matrix. All buses in the system are converted to nodes to be modeled. The total number of nodes for each bus is equal to the number of available or modeled phases at the bus. The nodal admittance matrix $[Y]$ is constructed based on the nodal admittance model for each impedance branch in the system. 
The constant impedance load and shunt compensation at the bus are treated as self admittances, to be embedded into the nodal admittance matrix.

Due to the dependency of nodal current injections on nodal voltages, the solution of Equation (1) is obtained through iteratively solving the following nodal current injection mismatch equations:

$$
[\Delta I]=[Y][\Delta V]
$$

Equation (2) only includes the nodes for PQ and PV buses. The nodes associated with the swing bus are not included, because their complex voltages are known. At each iteration, a current injection mismatch for each node $[\Delta I]$ is first determined based on the properties of connected loads and sources and the most recently computed nodal voltages. Then, an incremental voltage change $[\Delta V]$ is computed using the factorized nodal admittance matrix.

The initial voltages for each bus are set to the values at the swing bus multiplied by an aggregated voltage amplifying factor matrix introduced by the transformers or voltage regulators along the shortest path between the swing bus and the current bus:

$$
V_{p}^{(0)}=\prod_{s t} A_{V_{s t}} V_{\text {swing }}
$$

where, $V_{p}^{(0)}$ is the vector of initial complex voltages of bus $p, V_{\text {swing }}$ is the complex voltage of the swing bus, $A_{V_{s t}}$ is the voltage amplifying factor matrix of a regulator or transformer between the two buses, bus $s$ and bus $t$ residing on the shortest path from the swing bus to the bus under consideration.

The complex admittance matrix $\mathrm{Y}$ consists of two integrated parts, one real part and one imaginary part. The real part is a conductance matrix $\mathrm{G}$, and the imaginary part is a susceptance matrix B. Accordingly, the equation (2) can be rewritten in rectangular coordinates as:

$$
\left[\begin{array}{c}
\Delta I_{r} \\
\Delta I_{m}
\end{array}\right]=\left[\begin{array}{cc}
G & -B \\
B & G
\end{array}\right]\left[\begin{array}{l}
\Delta V_{r} \\
\Delta V_{m}
\end{array}\right]
$$

where, $\Delta I_{r}$ and $\Delta I_{m}$ are the real part and imaginary part of the nodal current injection mismatches, and $\Delta V_{r}$ and $\Delta V_{m}$ are the real part and imaginary part of the incremental nodal voltage changes. Both $\mathrm{G}$ and $\mathrm{B}$ matrices contribute to the changes of nodal voltages.

The effects of $G$ and $B$ matrices on nodal voltage changes are decoupled into two separate equations, and each set of equations considers the contribution of only one matrix. The final voltage change is formed as a weighted linear combination of the two contributions.

Ignoring the effects of $\mathrm{B}$ matrix, the nodal voltage changes resulting from $\mathrm{G}$ matrix can be determined as:

$$
\left[\begin{array}{l}
\Delta I_{r} \\
\Delta I_{m}
\end{array}\right]=\left[\begin{array}{ll}
G & \\
& G
\end{array}\right]\left[\begin{array}{l}
\Delta V_{r 1} \\
\Delta V_{m 1}
\end{array}\right]
$$

where $\Delta V_{r 1}$ and $\Delta V_{m 1}$ are the corresponding real and imaginary parts of the voltage changes resulting from matrix G. Hence, the first set of decoupled equations is given by:

$$
\begin{aligned}
& {\left[\Delta I_{r}\right]=[G]\left[\Delta V_{r 1}\right]} \\
& {\left[\Delta I_{m}\right]=[G]\left[\Delta V_{m 1}\right]}
\end{aligned}
$$

Ignoring the effects of matrix $\mathrm{G}$, the nodal voltage changes resulting from matrix $\mathrm{B}$ can be determined as:

$$
\left[\begin{array}{l}
\Delta I_{r} \\
\Delta I_{m}
\end{array}\right]=\left[\begin{array}{ll} 
& -B \\
B &
\end{array}\right]\left[\begin{array}{l}
\Delta V_{r 2} \\
\Delta V_{m 2}
\end{array}\right]
$$

where $\Delta V_{r 2}$ and $\Delta V_{m 2}$ are the corresponding real and imaginary parts of voltage changes resulting from matrix $B$. Similarly, the second set of decoupled equations is given by:

$$
\begin{gathered}
{\left[\Delta I_{m}\right]=[B]\left[\Delta V_{r 2}\right]} \\
{\left[\Delta I_{r}\right]=-[B]\left[\Delta V_{m 2}\right]}
\end{gathered}
$$

The net nodal voltage change is given by a weighted combination of the two decoupled changes:

$$
\left[\begin{array}{c}
\Delta V_{r} \\
\Delta V_{m}
\end{array}\right]=\mathrm{A}\left[\begin{array}{c}
\Delta V_{r 1} \\
\Delta V_{m 1}
\end{array}\right]+(I-\mathrm{A})\left[\begin{array}{c}
\Delta V_{r 2} \\
\Delta V_{m 2}
\end{array}\right]
$$

where $\mathrm{A}$ is a diagonal weight matrix that represents the contribution of matrix $G$ towards the whole voltage update, and $I$ is an identity matrix.

\section{B. Zero-impedance branches}

Many branches in a distribution system can be regarded as zero-impedance branches, such as step voltage regulators, switches, ideal transformers, very short lines and jumpers. Those branches have been merged with adjacent impedance branches to be modeled. Fig.1 shows an example of a distribution system with a generalized three-phase zeroimpedance branch.

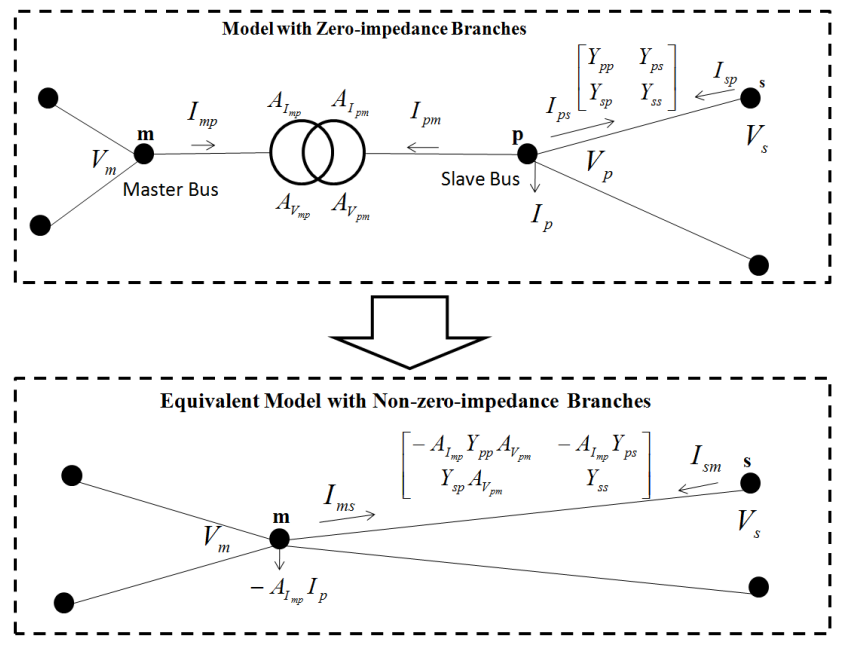

Fig.1. An Equivalent Model of Zero-impedance Branch

In the Fig. 1, the zero-impedance branch connects bus $m$ and bus $p$ with an ideal transformer. One of the buses, for example the bus $m$, is assigned to be a master bus, and the other bus $p$ is assigned to be a slave bus. The slave bus is connected with a load current $I_{p}$. The transformer's functionality is represented by two voltage amplifying factor matrices, $A_{V_{m p}}$ and $A_{V_{p m}}$, and two current amplifying factor matrices, $A_{I_{m p}}$ and $A_{I_{p m}}$. Those amplifying factor matrices are determined by the winding connection and tap positions for a transformer or a voltage regulator, and by the phase 
connection for a switch, a short line, or a jumper.

The relationship of bus voltages and branch currents of the zero-impedance branch can be described as:

$$
\begin{gathered}
I_{m p}=A_{I_{m p}} I_{p m} \\
I_{p m}=A_{I_{p m}} I_{m p} \\
V_{m}=A_{V_{m p}} V_{p} \\
V_{p}=A_{V_{p m}} V_{m}
\end{gathered}
$$

where, $I_{m p}$ and $I_{p m}$ are the phase currents flowing into the branch through the master bus $m$, and slave bus $p$ respectively, and $V_{m}$ and $V_{p}$ are the phase-to-ground voltages at the master bus and slave bus.

As shown in Fig. 1, the zero-impedance branch is merged into adjacent impedance branches, such that the slave bus is not considered in analysis of the model. In the example of Fig. 1, the zero-impedance branch is connected to two branches by the slave bus $p$, and to two other branches by the master bus $m$. Taking one adjacent branch between slave bus $p$ and bus $s$ as example, the relationship between the branch currents, and bus voltages for the branch can be described as:

$$
\left[\begin{array}{c}
I_{p s} \\
I_{s p}
\end{array}\right]=\left[\begin{array}{cc}
Y_{p p} & Y_{p s} \\
Y_{s p} & Y_{s s}
\end{array}\right]\left[\begin{array}{c}
V_{p} \\
V_{s}
\end{array}\right]
$$

where $I_{p s}$ and $I_{s p}$ are the vectors of phase currents flowing through bus $p$ and bus $s$ into the branch, respectively, $V_{p}$ and $V_{s}$ are the vectors of phase voltages at bus $p$ and bus $s$, $Y_{p p}$ and $Y_{s s}$ are the self admittance matrices at bus $p$ and bus $s$, and $Y_{p s}$ and $Y_{s p}$ are the mutual admittance matrices between bus $p$ and bus $s$, and bus $s$ and bus $p$, respectively.

In the equivalent model, the zero-impedance branch and the slave bus $p$ are removed. There are no changes for the branches connected to the master bus $m$. The branches connected to the slave bus $p$ are reconnected to bus $m$, and the branch admittance matrices and the current injections at the master bus $m$ are modified accordingly. The load current $I_{p}$ at bus $p$ is modeled as equivalent currents at bus $m$, using $-A_{I_{m p}} I_{p}$. The branch between bus $p$ and bus $s$ in the system is replaced with a new branch directly between bus $m$ and bus $s$, and the branch currents, $I_{m s}, I_{s m}$, and the nodal voltages, $V_{m}$ and $V_{s}$, are related as

$$
\left[\begin{array}{c}
I_{m s} \\
I_{s m}
\end{array}\right]=\left[\begin{array}{cc}
-A_{I_{m p}} Y_{p p} A_{V_{p m}} & -A_{I_{m p}} Y_{p s} \\
Y_{s p} A_{V_{p m}} & Y_{s s}
\end{array}\right]\left[\begin{array}{c}
V_{m} \\
V_{s}
\end{array}\right]
$$

The voltage amplifying matrix may be given in terms of line-to-line voltages for transformers with ungrounded connections. In this case, the following equation is used to convert the matrix from one based on given line-to-line voltages to one based on phase-to-ground voltages:

$$
A_{V_{m p}}=C_{V}^{P L} A_{V_{p m}}^{L L} C_{V}^{L P}
$$

where $A_{V_{p m}}^{L L}$ is the amplifying matrix associated with the line-to-line voltages of bus $p$ and bus $m$, and $C_{V}^{P L}$ is a voltage conversion matrix that is used to convert the line-toline voltages into phase-to-ground ones according to:

$$
C_{V}^{P L}=\left[\begin{array}{ccc}
1 / 3 & 0 & -1 / 3 \\
-1 / 3 & 1 / 3 & 0 \\
0 & -1 / 3 & 1 / 3
\end{array}\right]
$$

$C_{V}^{L P}$ is a voltage conversion factor matrix that is used to convert the phase-to-ground voltages into line-to-line voltages according to:

$$
C_{V}^{L P}=\left[\begin{array}{ccc}
1 & -1 & 0 \\
0 & 1 & -1 \\
-1 & 0 & 1
\end{array}\right]
$$

The definition of $C_{V}^{L P}$ is straightforward, but not the same as for $C_{V}^{P L}$. The conversion from line-to-line voltages into phase-to-ground voltages is not trivial. Due to unknown neutral-to-ground voltages, multiple results may be obtained based on the same line-to-line voltages. Equation (20) is proposed to uniquely convert the line-to-line voltages to the phase-to-ground voltages. The conversion expressed in equations (19) is accurate when the voltages only include positive and negative sequence components, and is a good approximation if the zero-sequence components are small enough.

\section{Three-phase PV buses with balanced voltages}

A PV bus in the distribution system can be modeled as three independent PV nodes if active power and voltage magnitude of each phase is regulated independently. However, if a PV bus is connected to a three-phase synchronous generator, the three-phases of the PV bus cannot be modeled separately, since the three phases of the generator are balanced, three phases must have same magnitude of voltages, and constant phase angle difference between each two phases such that phase $a$ always leads phase $b$ by 120 degrees, and lags phase $c$ by 120 degrees. The regulated parameters of those generators are the magnitude of balanced voltages, and the total active power of three phases. Such three-phase PV bus has merged into one single phase PV bus, to be modeled in power flow equations based on admittance matrices. Fig. 2 shows an example of a distribution system with three-phase ganged regulated PV buses.

In the Fig. 2, bus $p$ is a three-phase PV bus with balanced voltages. The total power generation of bus $p, S_{p}$ can be determined as:

$$
S_{p}=\sum_{x \in\{a, b, c\}} V_{p}^{x} I_{p}^{x^{*}}
$$

where, $V_{p}^{x}$ and $I_{p}^{x}$ are the phase-to-ground voltage and phase current for phase $x$, and $I_{p}^{x^{*}}$ is the conjugate of $I_{p}^{x}$.

The three phases $a, b$, and $c$ are combined as an equivalent single-phase $e$. The equivalent phase can be any phase. Taking phase $a$ as an example, the phase voltage for the equivalent phase of bus $p, V_{p}^{e}$ can be set as: 


$$
V_{p}^{e}=V_{p}^{a}
$$

where, $V_{p}^{a}$ is the phase voltage for phase $a$. The current at the equivalent phase $e, I_{p}^{e}$ can be calculated as the summation of all three phase currents after rotation to the selected phase, e.g., currents at phase $b$ and at phase $c$ are rotated 120 degrees and -120 degrees, respectively:

$$
I_{p}^{e}=R^{T} I_{p}
$$

where, $I_{p}$ is the vector of three phase currents, $R$ is a rotation vector defined as:

$$
R=\left[\begin{array}{lll}
1 & e^{j 120^{\circ}} & e^{-j 120^{\circ}}
\end{array}\right]^{T}
$$

$R^{T}$ is a transpose of $R$. The total power of three phases at bus $p$ can be replaced with the power at the equivalent phase:

$$
S_{p}=V_{p}^{e} I_{p}^{e^{*}}
$$

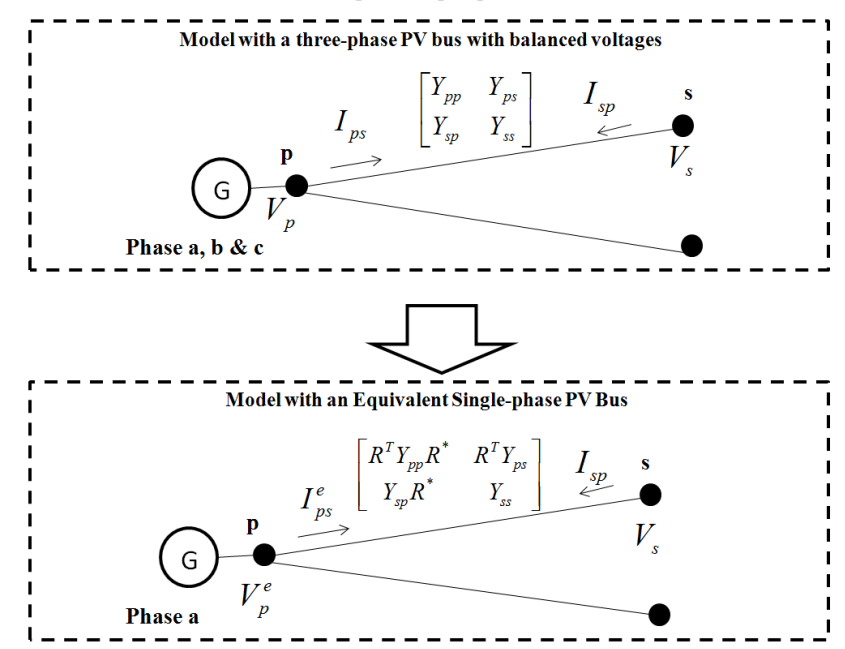

Fig.2. An Equivalent Model of Three-phase PV bus with balanced Voltages

The nodal admittance model for each branch connected with the three-phase PV bus needs to be modified accordingly. In Fig. 2, the three-phase PV bus $p$ is connected to two branches. Taking one branch between bus $p$ and bus $s$ as an example, the relationship between the phase currents of the branch and phase voltages of its terminal buses can be described by Equation (16). In the equivalent model, the three phases of PV buses with balanced voltages are combined into one single phase, and the new branch model can be described as:

$$
\left[\begin{array}{c}
I_{p s}^{e} \\
I_{s p}
\end{array}\right]=\left[\begin{array}{cc}
R^{T} Y_{p p} R^{*} & R^{T} Y_{p s} \\
Y_{s p} R^{*} & Y_{s s}
\end{array}\right]\left[\begin{array}{c}
V_{p}^{e} \\
V_{s}
\end{array}\right]
$$

\section{Equivalent Nodal Current Injections}

The calculation of nodal current injection mismatches depends on the associated bus type that itself is determined by the type of distribution loads, or the regulation type of equivalent sources or distribution generation sources connected to the bus.

The buses connected to constant power loads, or constant current loads, are modeled as PQ buses. A constant power load is specified by the active and reactive power injection at the bus. A constant current load is modeled as constant equivalent injected powers. The equivalent injected powers are based on estimated voltages, and are recalculated when the updated voltages become available during the iterations of the solution procedure. Equivalent sources, which represent the impacts of transmission systems on distribution systems, are modeled as swing buses or PV buses. Dependent on their regulation patterns, buses connected with distribution generators can be modeled as either PQ or PV buses. Buses connected to constant-powerfactor generators are treated as PQ buses, and buses connected to constant-voltage generators are treated as PV buses.

The equivalent current injection mismatch, $\Delta I_{i}^{x}$ of a PQ bus $i$ at phase $x$ can be determined from the scheduled power injections and the calculated equivalent current:

$$
\Delta I_{i}^{x}=S_{i}^{x-s c h^{*}} / V_{i}^{x(k)}-I_{i}^{x(k)} \quad x=\{a, b, c, e\}
$$

where $S_{i}^{x-s c h}$ is the scheduled complex power injections for bus $i$ at phase $x, V_{i}^{x(k)}$ is the voltage of bus $i$ at phase $x$ obtained at the latest iteration $k, I_{i}^{x(k)}$ is the equivalent current injections calculated from equation (1) based on the voltages at iteration $k$, and phase $x$ can be one of phase $a$, phase $b$, phase $c$, or the equivalent phase $e$. The scheduled active power and reactive power are given and kept constant during the iterations for a constant power load or a PQ type generation source, but are recalculated when the voltages are updated for a constant current load.

A PV bus uses the same equation (27) to determine the equivalent current injection mismatches. The scheduled active power is specified by the generation regulation specification. The scheduled reactive power is determined by an estimated power factor at the first iteration. The power factor can be a given rated power factor of the generator, or the average power factor of the system loads, if the rated power factor is not provided. The scheduled reactive powers for the iterations after the first one are updated from the difference between the scheduled and solved voltages. When a converged power flow is obtained, if the voltage magnitudes at PV nodes are not equal to the scheduled values, reactive powers are adjusted to maintain the scheduled voltages at the PV nodes.

The following equation is used to determine the required amount of compensation currents applied to the bus in order to eliminate the voltage magnitude mismatch:

$$
\left[\Delta I_{C}\right]=\left[Y_{C}\right]\left[\Delta V_{C}\right]
$$

where $\left[\Delta I_{C}\right]$ is the vector of compensated currents for the $\mathrm{PV}$ nodes, $\left[\Delta V_{C}\right]$ is the vector of required voltage changes, and $\left[Y_{C}\right]$ is a sensitivity matrix of nodal compensation currents with respect to nodal voltage changes for the PV nodes. The dimension of $\left[Y_{C}\right]$ is equal to the number of $\mathrm{PV}$ nodes. The sensitivity matrix is derived from the admittance matrix $[Y]$ in Equation (2) using a Kron reduction method to eliminate all elements related to all non-PV nodes. The compensation current vector contains one element for each three-phase ganged regulated PV bus, and three elements for each three-phase independent regulated PV bus.

Assuming that the phase angles of node voltage keep their values from the latest iteration $k$, the required voltage 
changes for a PV bus $i$ at phase $x, \Delta V_{C_{i}}^{x}$ can be determined as

$$
\begin{array}{r}
\Delta V_{C_{i}}^{x}=\left(\left|V_{i}^{x-s c h}\right|-\left|V_{i}^{x(k)}\right|\right) V_{i}^{x(k)} /\left|V_{i}^{x(k)}\right| \\
x=\{a, b, c, e\}
\end{array}
$$

where, $\left|V_{i}^{x-s c h}\right|$ is the scheduled voltage magnitude for bus $i$ at phase $x$.

By solving equation (28), approximated compensated currents can be determined to eliminate the voltage magnitude mismatch at the last iteration. Then, the required compensated reactive power at bus $i$ on phase $x, \Delta Q_{i}^{x-s c h}$, can be determined as:

$$
\Delta Q_{i}^{x-s c h}=\operatorname{Im}\left\{V_{i}^{x(k)} \Delta I_{C_{i}}^{x^{*}}\right\} \quad x=\{a, b, c, e\}
$$

where, $\Delta I_{C_{i}}^{x^{*}}$ is the conjugate of the compensated current at bus $i$ on phase $x$, and $\operatorname{Im}\{\bullet\}$ is the imaginary part of complex number. The required reactive power generation for a PV node is:

$$
Q_{i}^{x-s c h}=Q_{i}^{x-s c h(k)}+\beta_{i} \Delta Q_{i}^{x-s c h} \quad x=\{a, b, c, e\}
$$

where, $Q_{i}^{x-s c h(k)}$ is the scheduled reactive power calculated at the last iteration $k$, and $\beta_{i}$ is an accelerating factor to limit the reactive power changes within given maximum change, or the resulting reactive power generation within its capacity.

\section{E. G matrix contribution factors}

The selection of a contribution matrix of conductance or susceptance is crucial to the convergence performance of the method. The nodes in the system are divided into two groups, based on the relative magnitude of the corresponding diagonals of conductance and susceptance matrices, where one is the set of nodes for which the conductance is greater than susceptance, and the other is the set for which conductance is less than susceptance. A uniform contribution factor is used for each group. The maximum ratio of conductance to susceptance is used to set the contribution factor of the first group, and the minimum ratio is used for the second group. Only one uniform contribution factor is used if all nodes belongs to either the first or the second group.

The following computational procedure is used for determining the optimal value of the relative contribution factors, based on the diagonals of nodal conductance and susceptance matrices. Two control parameters, a lower ratio threshold $\underline{\alpha}$, e.g. 0.45 , and an upper ratio threshold $\bar{\alpha}$, e.g. 0.55 , are used to help determine the contribution factor for each node. The procedures includes:

(1) . Determining the contribution factor of $G$ matrix for each node based on the diagonals of $\mathrm{G}$ and $\mathrm{B}$ matrices at the corresponding row:

$$
\alpha_{i}=\left|g_{i i}\right| /\left(\left|g_{i i}\right|+\left|b_{i i}\right|\right)
$$

where $\alpha_{i}$ is the $\mathrm{G}$ contribution factor for the $i$-th row, and $g_{i i}$ and $b_{i i}$ are the $i$-th diagonal elements of $\mathrm{G}$ and $\mathrm{B}$ matrices respectively;

(2). Determining the lower and upper bounds for $G$ contribution factors according to:

$$
\begin{aligned}
& \alpha^{\max }=\max _{i} \alpha_{i} \\
& \alpha^{\min }=\min _{i} \alpha_{i}
\end{aligned}
$$

(3). Comparing the $G$ contribution factors for all nodes against the lower ratio threshold $\underline{\alpha}$. If all factors are greater than the lower threshold, the method proceeds to the next step (4). Otherwise, the method proceeds to step (5).

(4). Replacing the G contribution factor $\alpha_{i}$ of all nodes with the upper bound $\alpha^{\max }$;

(5). Comparing the $\mathrm{G}$ contribution factors for all nodes against the upper ratio threshold $\bar{\alpha}$. If all factors are less than the upper threshold, the method proceeds to the next step (6). Otherwise, the method proceeds to step (7);

(6). Replacing the G contribution factor $\alpha_{i}$ of all nodes with the lower bound $\alpha^{\text {min }}$;

(7). Counting the total number of nodes that $G$ matrix contribution is more than $B$ matrix, that is the $G$ contribution factor $\alpha_{i}$ is greater than 0.5 . If more nodes have more $\mathrm{G}$ contributions than $\mathrm{B}$, the method proceeds to the next step (8). Otherwise, the method proceeds to step (9);

(8). Replacing the $\mathrm{G}$ contribution factor $\alpha_{i}$ with the upper bound $\alpha^{\max }$ for all nodes that have a contribution factor greater than the lower ratio threshold $\underline{\alpha}$, and with the lower bounds $\alpha^{\text {min }}$ for the remaining rows;

(9). Replacing the $\mathrm{G}$ contribution factor $\alpha_{i}$ with the upper bound $\alpha^{\max }$ for all nodes that have a contribution factor greater than a upper ratio threshold $\bar{\alpha}$, and with the lower bounds $\alpha^{\mathrm{min}}$ for the remaining rows.

\section{NUMERICAL EXAMPLES}

The proposed method has been tested on several sample systems, including IEEE 37 node feeder, and IEEE 123 node feeder. Three different algorithms have been implemented to calculate the load flows of the sample systems, including the GB decoupled method proposed in this paper, the GB coupled equivalent current injection method that solves power flows through nodal current injection mismatch equations written in rectangular coordinates directly and without GB decoupling, and the Newton-Raphson method that solves power flows through nodal active and reactive power mismatch equations written in polar coordinates. The maximum mismatches of nodal active and reactive powers are set to be $10^{-5}$ per unit.

Table I and II gives the computational performances of the three algorithms on the IEEE 37 node test feeder, and IEEE 123 node test feeder, respectively. Both the computational time and total number of iterations are given to compare the performance of each algorithm. The computational procedure includes two stages, the first stage involves the construction of connected islands based on the current or study-mode switch status through topology analysis, and the second stage involves the calculation of 
load flows for each island that was constructed during the first stage.

TABLE I

COMPUTATIONAL PERFORMANCES ON IEEE 37 NODE FEEDER

\begin{tabular}{|c|c|c|c|}
\hline \multirow[b]{2}{*}{ Algorithm } & \multirow{2}{*}{$\begin{array}{c}\text { Number } \\
\text { of } \\
\text { Iterations }\end{array}$} & \multicolumn{2}{|c|}{ CPU Time $(\mathrm{ms})$} \\
\hline & & $\begin{array}{c}\text { Topology } \\
\text { Analysis }\end{array}$ & $\begin{array}{l}\text { Load Flow } \\
\text { Calculation }\end{array}$ \\
\hline Newton-Raphson & 6 & 0.166 & 62.780 \\
\hline GB Coupled & 5 & 0.166 & 36.944 \\
\hline GB Decoupled & 25 & 0.166 & 31.360 \\
\hline \multicolumn{4}{|c|}{$\begin{array}{c}\text { TABLE II } \\
\text { COMPUTATIONAL PERFORMANCES ON IEEE } 123 \text { NODE FEEDER }\end{array}$} \\
\hline \multirow[b]{2}{*}{ Algorithm } & \multirow{2}{*}{$\begin{array}{c}\text { Number } \\
\text { of } \\
\text { Iterations }\end{array}$} & \multicolumn{2}{|c|}{ CPU Time $(\mathrm{ms})$} \\
\hline & & $\begin{array}{l}\text { Topology } \\
\text { Analysis }\end{array}$ & $\begin{array}{l}\text { Load Flow } \\
\text { Calculation }\end{array}$ \\
\hline Newton-Raphson & 24 & 0.633 & 294.274 \\
\hline GB Coupled & 17 & 0.633 & 188.601 \\
\hline GB Decoupled & 65 & 0.633 & 102.098 \\
\hline
\end{tabular}

Taking the results from the IEEE 37 node feeder as an example, each algorithm used the same amount of time, $0.166 \mathrm{~ms}$ to complete its topology analysis, but the total number of iterations and load flow calculation time are different for the three different algorithms. It took $31.360 \mathrm{~ms}$ and 25 iterations for the proposed algorithm to find the final solution with the required precision. In comparison, it took $62.780 \mathrm{~ms}$ and 6 iterations for the Newton-Raphson algorithm, and $36.944 \mathrm{~ms}$ and 5 iterations for the GB coupled algorithm to find the solution with the same precision. Although taking more iterations to find the solution, the proposed algorithm is more efficient compared with the Newton-Raphson method and the GB coupled equivalent current injection method. Similar results can be found in Table II. Despite of the difference of computation time, the results obtained by three different algorithms are exactly the same. Table III gives the calculated voltage results for a set of sample nodes of the 37 node system.

TABLE III

RESULTS OF NODE VOLTAGES FOR IEEE 37 NODE FEEDER

\begin{tabular}{c|c|c|c}
\hline \multirow{2}{*}{$\begin{array}{c}\text { Node } \\
\text { Name }\end{array}$} & \multicolumn{3}{|c}{ Phase-to-phase Voltage (p.u. @ deg.) } \\
\cline { 2 - 4 } & A-B & B-C & C-A \\
\hline 701 & $1.0317 @-0.08$ & $1.0144 @-120.39$ & $1.0183 @ 120.61$ \\
\hline 720 & $1.0205 @-0.21$ & $1.0011 @-120.66$ & $1.0040 @ 120.53$ \\
\hline 740 & $0.9981 @ 0.08$ & $0.9961 @-120.75$ & $0.9846 @ 119.76$ \\
\hline
\end{tabular}

Table IV shows the computational performance of the proposed algorithm on the IEEE 123 test feeder with PV buses. The first test scenario is the 123 node feeder which has no PV buses. It is used as a base for comparing with other scenarios with different number of PV buses. With the increase in the number of PV buses, the required number of iterations and computational time are significantly increased. The required number of iterations is heavily dependent on the estimation accuracy of the initial reactive power generations. The more accurate the estimation accuracy, the smaller number of iterations are required.

TABLE IV

COMPUTATIONAL PERFORMANCES ON VARIOUS SCENARIOS WITH PV BUSES

\begin{tabular}{|c|c|c|c|c|}
\hline \multicolumn{2}{|r|}{ Test Scenarios } & \multirow{2}{*}{$\begin{array}{c}\text { Number } \\
\text { of } \\
\text { Iterations }\end{array}$} & \multicolumn{2}{|c|}{ CPU Time(ms) } \\
\hline $\begin{array}{c}\text { Number } \\
\text { of PV } \\
\text { buses }\end{array}$ & $\begin{array}{c}\text { Nodes modeled as PV } \\
\text { buses }\end{array}$ & & $\begin{array}{l}\text { Topology } \\
\text { Analysis }\end{array}$ & $\begin{array}{l}\text { Load Flow } \\
\text { Calculation }\end{array}$ \\
\hline 0 & 1 & 65 & 0.633 & 102.98 \\
\hline 2 & 66,88 & 2219 & 0.656 & 2163.901 \\
\hline 4 & $30,56,66,88$ & 7583 & 0.660 & 7369.812 \\
\hline 8 & $\begin{array}{c}18,25,30,42,56,66,72 \\
88\end{array}$ & 35316 & 0.693 & 35637.341 \\
\hline
\end{tabular}

\section{CONCLUSION}

A three-phase load flow method has been proposed by formulating the power flow problem by means of nodal equivalent current injection equations in rectangular coordinates. The voltage changes resulting from nodal admittance matrices are decoupled into one contribution from the real part, the conductance matrix, and another contribution from the imaginary part, the susceptance matrix. The method determines the voltage changes resulting from conductance and susceptance matrices respectively, and determines the voltages of a node as a liner combination of those voltage changes. The relative contribution of a conductance or reactance matrix is determined based on the diagonals of conductance and susceptance matrices.

The PV nodes have been converted into PQ nodes by means of a sensitivity matrix determined through Kron reduction of the nodal admittance matrix, and the corresponding reactive powers are adjusted after each solution has converged.

The proposed method models accurately the impacts of zero-impedance branches and three-phase voltage-balanced PV buses. Zero-impedance branches are merged with adjacent impedance branches, and the three phases of a balanced-voltage PV bus are merged into one single-phase PV bus to be modeled in the nodal admittance matrix.

Compared with the Newton-Raphson method and the rectangular coordinate equivalent current injection method, the proposed method requires relatively more iterations, but much less time to get to the solution.

\section{REFERENCES}

[1] W. H. Kersting, "A method to the design and operation of a distribution system", IEEE Transactions on Power Apparatus and Systems, vol. PAS-103, no.7, pp. 1945-1952, July 1984.

[2] D. Shirmohammadi, H. W. Hong, A. Semlyen, and G. X. Luo, "A Compensation-based Power Flow Method for Weakly Meshed Distribution and Transmission Networks", IEEE Transactions on Power Systems, vol. 3, no. 2, pp. 753-762, May 1988.

[3] G.X. Luo and A. Semlyen, "Efficient Load Flow for Large Weakly Meshed Networks", IEEE Transactions on Power Systems, vol. 5, no. 4, pp. 1309-1316, November 1990.

[4] W.C. Wu, and B.M. Zhang, "A three-phase power flow algorithm for distribution system power flow based on loop-analysis method", Electrical Power and Energy Systems, vol. 30, pp. 8-15, June 2007.

[5] T.-H. Chen, M.-S. Chen, K.-J Hwang, P. Kotas, and E.A. Chebli, "Distribution system power flow analysis-a rigid approach," IEEE Transactions on Power Delivery, vol.6, no.3, pp.1146-1152, Jul 1991.

[6] J. H. Teng, "A Modified Gauss-Seidel algorithm of three-phase power flow analysis in distribution network", Electrical Power and Energy Systems, vol. 24, no. 2, pp. 97-102, Feb. 2002.

[7] V. M. da Costa, N. Martins, and J.L.R. Pereira, "Developments in the Newton Raphson power flow formulation based on current injections", IEEE Transactions on Power Systems, vol.14, no.4, pp.1320-1326, November 1999.

[8] P. A. N. Garcia, J. L. R. Pereira, S. Carneiro Jr., V. M. da Costa, and N. Martins, "Three-phase power flow calculations using the current injection method", IEEE Transactions on Power Systems, vol.15, no.2, pp.508-514, May 2000.

[9] Whei-Min Lin, and Jen-Hao Teng; "Three-phase distribution network fast-decoupled power flow solutions", International Journal of Electrical Power \& Energy Systems, vol. 22, no. 5, pp. 375-380, June 2000.

[10] M. Abdel-Akher, K.M. Nor, and A.H.A. Rashid; "Improved ThreePhase Power-Flow Methods Using Sequence Components," IEEE Transactions on Power Systems, vol.20, no.3, pp. 1389- 1397, August 2005. 